\title{
Organophosphate intermediate syndrome with neurological complications of extrapyramidal symptoms in clinical practice
}

\author{
Mark B. Detweiler ${ }^{1,2,3}$ \\ ${ }^{1}$ Psychiatry Service, ${ }^{3}$ Geriatric Research Group, Veterans Affairs Medical Center, Salem, Virginia, ${ }^{2}$ Department of Psychiatry and Behavioral \\ Medicine, Virginia Tech-Carilion School of Medicine, Roanoke, VA, USA
}

\section{ABSTRACT}

Organophosphates (OPs) are ubiquitous in the world as domestic and industrial agricultural insecticides. Intentional poisoning as suicides attempts are clinical phenomena seen in emergency departments and clinics in agricultural areas. Intermediate syndrome with the neurological complication of extra pyramidal symptoms following acute OP ingestion may occur in pediatric and adult cases. While death is the most serious consequence of toxic OP doses, low levels of exposure and nonfatal doses may disrupt the neurobehavioral development of fetuses and children in addition to bring linked to testicular cancer and male and female infertility. These are disturbing. Chronic and acute toxicity from OPs are barriers to the health of our present and future generations. Symptoms and treatment of acute and chronic OP exposure are briefly referenced with inclusion of the intermediate syndrome. Suggestions for local and systemic reduction of the acute and long term consequences of OP ingestion are opined.

Key words: Organophosphates, intermediate syndrome, suicide, poisoning, adolescent

\section{Introduction}

Organophosphates (OPs) include a variety of formulations utilized for multiple purposes, most frequently as domestic and industrial agricultural insecticides, herbicides, fungicides, and rodenticides ${ }^{[1]}$ Many OPs are highly toxic to plants, animals, and humans. ${ }^{[2]}$ During metabolism, approximately $75 \%$ of OPs are converted into metabolites called dialkyl-phosphate metabolites that cause some OP neurotoxicity at chronic doses. ${ }^{[3]}$ OPs act as neurotoxins by inhibiting acetylcholinesterase in the central and peripheral nervous system, producing excess acetylcholine (ACh) and multiple neurological symptoms. ${ }^{[4,5]}$ Even at very low levels of exposure, OPs cause disruption in the neurobehavioral development of fetuses and children. ${ }^{[1]}$ Chronic OP exposure has

\begin{tabular}{|l|l|}
\hline \multicolumn{2}{|c|}{ Access this article online } \\
\hline Quick Response Code: & Website: \\
\hline & www.ruralneuropractice.com \\
\cline { 2 - 3 } & \\
\hline
\end{tabular}

been linked to testicular cancer and male and female infertility. ${ }^{[6]}$

The featured case report, "Organo-phosphorus phosphorus-induced extrapyramidal intermediate syndrome in an adolescent suicide attempt survivor" by Doctors Sarkar, Nandi, Mondal, and Mandal ${ }^{[7]}$ regarding an OP suicide attempt by a 12-year-old girl, is an important contribution to the OP toxicity literature. Although the syndrome is relatively rare in acute OP intoxication, with an estimated number of less than 30 cases reported in the medical literature, the incidence in worldwide clinical practice is probably much higher. The case report is instructive for several reasons. First, it reminds treatment teams and rural practitioners that OP intermediate syndrome with the neurological complication of extrapyramidal symptoms following acute OP ingestion may occur in pediatric cases. Second, it has short- and long-term sequelae, particularly for female children and adolescents: death ${ }^{[8]}$ damage to the maturing nervous and endocrine systems; ${ }^{[9]}$ disturbances of behavior and long-term mental health; ${ }^{[4,10]}$ and future birth defects or infertility. ${ }^{[11]}$ These are disturbing barriers to the health of our present and future generations. It also reminds us of the need to protect our children

Address for correspondence:

Dr. Mark B. Detweiler, MD (116A7), Veterans Affairs Medical Center, 1970 Roanoke Boulevard, Salem, Virginia, 24153, USA.

E-mail: Mark.Detweiler1@va.gov 
and young adults from indiscriminant access to OPs and their misuse for suicide attempts. In nonmedical environments, keeping OPs in secured storage areas may reduce access to those persons who wish to use them for suicide attempts and from inadvertent exposure. ${ }^{[12,13]}$

\section{Acute organophosphate exposure}

Suicide attempts employing OPs is a frequent presentation in emergency departments (EDs), critical care units, and medicine wards ${ }^{[10]}$ in agricultural areas of the world,,$^{[14]}$ especially where there is a prohibition of having firearms. ${ }^{[15]}$ Symptom onset of acute OP intoxication is rapid following cutaneous absorption, inhalation, oral ingestion or injection, with severity related to the specific compound, amount, route of exposure, and rate of metabolic degradation. ${ }^{[16,17]}$ Acute OP exposure results in a rapid and severe neural and respiratory crisis. ${ }^{[18,19]}$ The morbidity and mortality associated with OP intoxication is related to the compound's action at the muscarinic, nicotinic receptors, and the central nervous system. ${ }^{[19]}$

Acute OP intoxication may be suggested by depressed levels of plasma pseudocholinesterase $(\geq 20 \%)$ and/or red blood cell acetylcholinesterase $(\geq 15 \%)$, indicating excessive OP absorption. In pediatric cases, the diagnosis may be confounded as the nonspecific symptoms of acute OP poisoning may be misdiagnosed as common pediatric illnesses, such as respiratory infections, viral syndromes, gastroenteritis, atopic dermatitis, or drug-related encephalopathy. ${ }^{[17]}$ Seizures, uncommon in adult cases of OP poisoning, are seen more frequently in pediatric cases and may be a diagnostic clue in the early differential diagnosis. ${ }^{[20]}$

Acute OP intoxication syndrome onset may vary from almost instantaneous to several hours after exposure. The three phases of neurological illness following OP intoxication are well reported. Phase I (acute cholinergic crisis) occurs secondary to continued depolarization at the neuromuscular junction. Phase II (intermediate syndrome) develops 24-96 hours after phase I resolution. It is characterized by weakness of respiratory muscles, proximal muscles, and cranial nerve palsies. Extrapyramidal symptoms are uncommon, but can be a serious occurrence in OP poisoning in Phase II. ${ }^{[21]}$ Phase III (organophosphate-induced delayed polyneuropathy) presents 2-3 weeks after OP exposure. ${ }^{[22]}$ Evidence suggests that toxic OP blood levels precipitate extrapyramidal features due to the OP effect on the basal ganglia bilaterally. ${ }^{[23]}$ The extrapyramidal symptoms have been recorded to appear from day $4^{[24]}$ to day $40^{[21]}$ after OP exposure. Subsequent glutaminergic excitation in the subthalamic nucleus and decreased cortical glutamate stimulation may reduce striatal activity causing decreased cortical glutamate stimulation. This may precipitate a dopamine-deficiency-like clinical presentation consisting of marked Parkinsonism, including bradyphrenia, rigidity, resting tremors, akinesia, and speech impairment. ${ }^{[19,25]}$ Regrettably, the Parkinsonism features may be misdiagnosed as acute reactive depression or psychosis ${ }^{[2]}$ leading to inappropriate pharmacotherapy and prolonged syndrome duration with increased risk of morbidity and mortality.

Medical treatment for acute OP poisoning should include airway control to insure adequate oxygenation. In cases of acute respiratory distress due to laryngospasm, bronchospasm, bronchorrhea, or seizures, intubation may be required. Intubation may be avoided by aggressive use of atropine. Use of succinylcholine is not recommended as it is degraded by plasma cholinesterase and may prolong paralysis. Benzodiazepines may be utilized for seizure control. Specific antidotes include atropine (muscarinic receptor blocker) to block the organophosphate-induced overstimulation of central and muscarinic cholinergic nerve terminals and pralidoxime (2-PAM), a cholinesterase reactivator (oxime) that assists in restoring respiratory and skeletal muscle strength. The latter does not reverse central effects as it does not cross the blood-brain barrier. ${ }^{[18]}$

Treatment with amantadine may weakly antagonize NMDA-type glutamate receptors with subsequent increase of glutamate excitation in the subthalamic nucleus fostering inhibited ACh release in striatal interneurons. Investigations of medications to reduce the toxicity of soman (OP nerve gas) on the central nervous system has been demonstrated in rats. Raveh et al. ${ }^{[27]}$ reported that when used prophylactically, caramiphen prevented the NMDA-induced lethality of soman. The authors suggested that effect of caramiphen against OP toxicity may be related to its capacity to modulate NMDA receptors in addition to its anticholinergic properties. ${ }^{[27]}$ In one case amantadine $100 \mathrm{mg}$ three times a day appeared to increase dopamine release by blocking dopamine reuptake with symptom alleviation. ${ }^{[19]}$

An ECG should be performed with continuous cardiac monitoring and pulse oximetry should be ongoing. ${ }^{[17]}$ A large study by Paul and Bhattacharyya evaluated the electrocardiographic changes in 107 patients with acute OP poisoning. Many cardiac irregularities were recorded: Prolonged Q-Tc (62.6\%); sinus tachycardia (33.6\%); sinus bradycardia (30.8\%); elevation of ST segment $(25.2 \%)$; T wave inversion (19.6\%); first-degree heart block (8.4\%); atrial fibrillation (4.6\%); ventricular tachycardia (5.6\%); and ventricular premature complexes $(2.8 \%) .{ }^{[28]}$ 
Death occurred from ventricular fibrillation and non-cardiogenic pulmonary edema in 17 (15.9\%) of the 107 patients with OP poisoning. The authors concluded that all patients with acute organophosphorus poisoning should be carefully monitored by ECG and depending clinical and biochemical parameters be transferred to the ICU to reduce OP poisoning mortality. ${ }^{[28]}$

Intravenous magnesium sulfate has been reported as beneficial for organophosphate toxicity. ${ }^{[29]}$ Isotonic sodium chloride solution or lactated Ringer's solution may be employed to irrigate the eyes of patients who have had ocular exposure to OPs. Morgan lenses can be used for eye irrigation. ${ }^{[17]}$

\section{Chronic organophosphate exposure}

Health care providers must avoid contaminating themselves while handling patients with OP toxicity. Protective equipment (e.g., neoprene gloves and gowns) is recommended when decontaminating patients with OP toxicity as hydrocarbons can penetrate nonpolar substances such as latex and vinyl. Clothing contaminated with OPs is considered hazardous waste and should be treated as such. Clinician respiratory protection when decontaminating OP patients who are significantly contaminated requires charcoal cartridge masks.

Continuous exposure to OPs, such as often occurs with agricultural workers or through contaminated food, may lead to cases of chronic OP toxicity. Protective clothing should be worn when employing OPs. Upon finishing OP application, clothes and skin need to be cleansed with warm water and soap. Screening for chronic OP toxicity is accomplished by using a urine test $(5 \mathrm{~mL}$ urine, high-performance thin layer chromatography) that quantifies six of OP dialkyl phosphates metabolites: dimethylphosphate dimethylthiophosphate dimethyldithiophosphate diethylphosphate diethylthiophosphate and diethyldithiophosphate.

Treatment for the chronic effects of OPs includes detoxification supports. ${ }^{[1]}$ Ensuring adequate consumption of docosahexanoic acid, an omega-3 fatty acid found in fish, such as salmon, tuna, bluefish, mackerel, swordfish, anchovies, herring, and sardines, may also help to increase antioxidant activity in the brain and prevent organophosphates-induced damage. ${ }^{[30]}$ Antioxidant nutrients, including vitamin E, vitamin C, and alpha-lipoic acid, may also protect against organophosphate-induced oxidative stress. ${ }^{[31,32]}$ The organic burden of organophosphates can be reduced with supplemental nutrients to stimulate detoxification (e.g., taurine, glycine, and $\mathrm{N}$-acetylcysteine). ${ }^{[11]}$

In the United States, diet is the primary route of exposure. ${ }^{[4]}$ However, the Food Quality Protection Act (FQPA) of 1996 markedly improved the safety standards that Environmental Protection Agency (EPA) uses in evaluating potential pesticide risks, especially for children. Since 1996, EPA has reviewed and reassessed 9721 tolerances (maximum permitted residues) for all pesticides involved in consumer foods. The EPA reviewed the risk of 613 pesticides first registered in the United States before November 1984, specifically regarding the effect on infants and children. As a result of this assessment, 21 of 49 OP pesticides registered at the beginning of EPA's review have been voluntarily canceled or are being phased out of use in the United States. Consequently, OP pesticide poisonings were reduced by $70 \%$ from 1994 to 2004 . Moreover, the amount of OP pesticides used in children's foods decreased from 28 million pounds per year to 12 million pounds per year. ${ }^{[33]}$

\section{Summary}

OP intoxication can be life-threatening, whether it is precipitated by accidental exposure, particularly in small children and adolescents, or by suicide attempts. It has been suggested that to highlight the role of OPs exclusively as agents for suicide overemphasizes the responsibility for prevention to individuals and underemphasizes the governmental, educational, and corporate responsibility of instituting safety policies and educational programs to reduce to risk of OP exposure and suicide attempts. ${ }^{[14]}$ Understanding that the OP intermediate syndrome with the neurological complications of extrapyramidal symptoms may complicate the diagnosis and treatment of pediatric OP intoxication merits discussion among medical students, medicine residents, ED residents, and primary care physicians in rural practice in addition to all ER staffs where OP intoxication cases may be anticipated. The comprehensive and diligent supervision of children and adolescents is not possible in many cases. The primary protective measure to avoid both $\mathrm{OP}$ intoxication and chronic $\mathrm{OP}$ is to establish national policies, comprehensive regulations, and education for the safe handling and storage of OPs for domestic, agriculture, and industrial use.

\section{References}

1. Diamanti-Kandarakis E, Bourguignon JP, Giudice LC, Hauser R, Prins GS, Soto AM, et al. Endocrine-disrupting chemicals: An endocrine society scientific statement. Endocr Rev 2009;30:293-342. 
2. Clothianidin - Registration Status and Related Information. U.S. EPA. 27 July 2012.

3. Slotkin TA. Does early-life exposure to organophosphate insecticides lead to prediabetes and obesity? Reprod Toxicol 2011;31:297-301.

4. Bouchard MF, Bellinger DC, Wright RO, Weisskopf MG. Attention-deficit/hyperactivity disorder and urinary metabolites of organophosphate pesticides. Pediatrics 2010;125:e1270-7.

5. Center for Disease Control. Organophosphorus insecticides: Dialkyl phosphate metabolites. National Report on Human Exposure to Environmental Chemicals-Fact Sheet. 2011. Available from: http://www. cdc.gov/exposurereport/OP-DPM_FactSheet.html. [Last accessed on 2014 Jan 17].

6. Center for Disease Control. National Survey of Family Growth. 2011. Available from: http://www.cdc.gov/nchs/nsfg.htm. [Last accessed on 2014 Jan 17].

7. Sarkar S, Nandi M, Mondal R, Mandal SK. Organo-phosphorus phosphorus-induced extra pyramidal intermediate syndrome in an Adolescent suicide attempt survivor. J Neurosci Rural Pract 2014;5:276-8.

8. Weissmann-Brenner A, David A, Vidan A, Hourvitz A. Organophosphate poisoning: A multihospital survey. Isr Med Assoc J 2002;4:573-6.

9. Sisk CL, Zehr JL. Pubertal hormones organize the adolescent brain and behavior. Front Neuroendocrinol 2005;26:163-74.

10. Singh S, Sharma N. Neurological syndromes following organophosphate poisoning. Neurol India 2000;48:308-13.

11. Redmond E, Egeston C, Bralley JA. Low level prenatal exposure to organophosphate pesticides significantly lowers IQ in children. Townsend Letter. Jan 2012.

12. Guidelines for the safe use of pesticides in non-agricultural workplaces. Available from: http://www.public.health.wa.gov.au/cproot/2158/2/ Guidelines for the safe use of pesticides in non-agricultural workplaces. pdf [Last accessed on 2013 Dec 19].

13. Code of practice for the safe use and storage of chemicals (including pesticides and herbicides) in agriculture. Available from: http://www. workcover.nsw.gov.au/formspublications/publications/Documents/ chemicals_in_agriculture_safe_use_storage_code_practice_0422. pdf [Last accessed on 2013 Dec 12].

14. London L, Flisher AJ, Wesseling C, Mergler D, Kromhout H. Suicide and exposure to organophosphate insecticides: Cause or effect? Am J Indian Med 2005;47:308-21.

15. Ghaleiha A, Afzali S, Bazyar M, Khorsand F, Torabian S. Characteristics of hospitalized patients following suicide attempt in Hamadan District, Iran. Oman Med J 2012;27:304-9.

16. Yurumez Y, Durukan P, Yavuz Y, Ikizceli I, Avsarogullari L, Ozkan S, et al. Acute organophosphate poisoning in university hospital emergency room patients. Intern Med 2007;46:965-9.

17. Katz KD. Organophosphate Toxicity Treatment and Management. Medscape 2013. Available from: http://emedicine.medscape.com/ article/167726-treatment [Last accessed on 2014 Jan 21].

18. Organophosphate pesticides and public health. 2007 Pediatric Environmental Health Specialty Unit (PEHSU), Department of Environmental and Occupational Health Sciences University of
Washington, opchild@u.washington.edu. [Last accessed on 2014 Jan 05].

19. Kalyanam B, Narayana S, Kamarthy P. A rare neurological complication of acute organophosphorous poisoning. Toxicol Int 2013;20:189-91.

20. Reigart JR, Roberts JR. Recognition and management of pesticide poisonings, $5^{\text {th }}$ ed, 1999.

21. Senanayake N, Sanmuganathan PS. Extrapyramidal manifestations complicating organophosphorous insecticide poisoning. Hum Exp Toxicol 1995;14:600-4.

22. Tanima D, Maisnam I, Kundu AK, Saha SP, Ghosh S, Maity A. Atypical central nervous system involvement in acute organophosphorus poisoning. J Assoc Physicians India 2011;59:326-7.

23. Liu H, Yang Y, Yang J, Meng L. Brain injury due to acute organophosphate poisoning: Magnetic resonance imaging manifestation and pathological characteristics. Neural Regen Res 2007;2:403-7.

24. Hsieh BH, Deng JF, Ger J, Tsai WJ. Acetylcholinesterase inhibition and the extrapyramidal syndrome: A review of the neurotoxicity of organophosphate. Neurotoxicology 2001;22:423-7.

25. Müller-Vahl KR, Kolbe H, Dengler R. Transient severe parkinsonism after acute organophosphate poisoning. J Neurol Neurosurg Psychiatry 1999;66:253-4.

26. Shahar E, Bentur Y, Bar-Joseph G, Cahana A, Hershman E. Extrapyramidal parkinsonism complicating acute organophosphate insecticide poisoning. Pediatr Neurol 2005;33:378-82.

27. Raveh L, Chapman S, Cohen G, Alkalay D, Gilat E, Rabinovitz I, et al. The involvement of the NMDA receptor complex in the protective effect of anticholinergic drugs against soman poisoning. Neurotoxicology 1999:20:551-9.

28. Paul UK, Bhattacharyya AK. ECG manifestations in acute organophosphorus poisoning. J Indian Med Assoc 2012;110:98,107-8.

29. Pajoumand A, Shadnia S, Rezaie A, Abdi M, Abdollahi M. Benefits of magnesium sulfate in the management of acute human poisoning by organophosphorus insecticides. Hum Exp Toxicol 2004;23:565-9.

30. Imesery ME, Al-Gayyar MM, Salem HA, Darweish MM, El-Mowafy A. Chemopreventive and renal protective effects for docosahexaenoic acid (DHA): Implications of CRP and lipid peroxides. Cell Div 2009;4:6.

31. Crinnion WJ. Environmental medicine, part 4: Pesticides-biologically persistent and ubiquitous toxins. Altern Med Rev 2000;5:432-47.

32. Al-Attar AM. Physiological and histopathological investigations on the effects of alpha-lipoic acid in rats exposed to malathion. J Biomed Biotechnol 2010;1-8.

33. Environmental Protection Agency. Setting Stricter Standards to Protect Infants and Children from Pesticide Risks. Available from: http://www. epa.gov/pesticides/health/children-standards.html [Last accessed on 2013 Dec 21].

How to cite this article: Detweiler MB. Organophosphate intermediate syndrome with neurological complications of extrapyramidal symptoms in clinical practice. J Neurosci Rural Pract 2014;5:298-301.

Source of Support: This material is the result of work supported with resources and the use of facilities at the Salem, Virginia Veterans Affairs Medical Center. Conflict of Interest: None declared. 